\title{
Stability of functional equations connected with quadrature rules
}

\section{Tomasz Szostok}

Dedicated to Professor Roman Ger on the occasion of his 70th birthday

Abstract. We study the stability properties of the equation

$$
F(y)-F(x)=(y-x) \sum_{i=1}^{n} a_{i} f\left(\alpha_{i} x+\beta_{i} y\right)
$$

which is motivated by numerical integration. In Szostok and Wassowicz (Appl Math Lett 24(4):541-544, 2011) the stability of the simplest equation of the type (0.1) was investigated thus the inequality

$$
|F(y)-F(x)-(y-x) f(x+y)| \leq \varepsilon
$$

was studied. In the current paper we present a somewhat different approach to the problem of stability of (0.1). Namely, we deal with the inequality

$$
\left|\frac{F(y)-F(x)}{y-x}-\sum_{i=1}^{n} a_{i} f\left(\alpha_{i} x+\beta_{i} y\right)\right| \leq \varepsilon .
$$

Mathematics Subject Classification. 39B82, 39B22, 65Q20.

Keywords. Stability of functional equations, functional equations stemming from numerical integration.

\section{Introduction}

In this paper we study the stability properties of the equation

$$
F(y)-F(x)=(y-x) \sum_{i=1}^{n} a_{i} f\left(\alpha_{i} x+\beta_{i} y\right) .
$$

Equation (1.1) is a profound generalization of the well known Aczél equation

$$
F(y)-F(x)=(y-x) f\left(\frac{x+y}{2}\right),
$$

which was motivated by the Lagrange mean value theorem (see [1]). 
Equation (1.1) was inspired by the quadrature rules of numerical integration. Functional equations inspired by numerical integration were studied among others in [3-9].

It can be proved (under some assumptions) that solutions of (1.1) are polynomial functions (see [4]). By a polynomial function of order $n$ we mean any solution of the functional equation $\Delta_{h}^{n+1} f(x)=0$, where $\Delta_{h}^{n}$ stands for the $n$th iterate of the difference operator $\Delta_{h} f(x)=f(x+h)-f(x)$.

The stability of (1.2) was studied in [10] where the inequality

$$
|F(y)-F(x)-(y-x) f(x+y)| \leq \varepsilon
$$

was considered.

In the current paper we present a somewhat different approach to the stability of (1.1). Equation (1.2) is known as the Aczél equation and was inspired by the Lagrange mean value theorem. Therefore it is natural to write (1.2) in the form

$$
\frac{F(y)-F(x)}{y-x}=f\left(\frac{x+y}{2}\right), x \neq y .
$$

Now, we may consider the following inequality

$$
\left|\frac{F(y)-F(x)}{y-x}-f\left(\frac{x+y}{2}\right)\right| \leq \varepsilon .
$$

Moreover, we shall study in this setting the stability properties of the more general equation (1.1).

\section{Results}

First we prove a technical lemma concerning a pexiderized version of (1.1)

$$
\frac{F(y)-F(x)}{y-x}=\sum_{i=1}^{n} f_{i}\left(\alpha_{i} x+\beta_{i} y\right) .
$$

Lemma 1. Let $n \in \mathbb{N}$ and let $\alpha_{i}, \beta_{i} \in \mathbb{R}, i=1, \ldots, n$. If functions $F, f_{1}, \ldots, f_{n}$ : $\mathbb{R} \rightarrow \mathbb{R}$ satisfy the inequality

$$
\left.\mid \frac{F(y)-F(x)}{y-x}-\sum_{i=1}^{n} f_{i}\left(\alpha_{i} x+\beta_{i} y\right)\right) \mid \leq \varepsilon
$$

then $f_{1}, \ldots, f_{n}$ satisfy the inequality

$$
\begin{aligned}
& \mid \sum_{i=1}^{n} f_{i}\left(\left(\alpha_{i}+\beta_{i}\right) x+\beta_{i} h\right)+\sum_{i=1}^{n} f_{i}\left(\left(\alpha_{i}+\beta_{i}\right) x+\left(\alpha_{i}+2 \beta_{i}\right) h\right) \\
& \left.\quad-2 \sum_{i=1}^{n} f_{i}\left(\left(\alpha_{i}+\beta_{i}\right) x+2 \beta_{i} h\right)\right) \mid \leq 4 \varepsilon .
\end{aligned}
$$


Proof. Taking $x+h$ instead of $y$ in (2.2), we get

$$
\left|\frac{F(x+h)-F(x)}{h}-\sum_{i=1}^{n} f_{i}\left(\left(\alpha_{i}+\beta_{i}\right) x+\beta_{i} h\right)\right| \leq \varepsilon,
$$

further, taking $x+2 h, x+h$ in place of $y, x$ resp. in (2.2), we get

$$
\left|\frac{F(x+2 h)-F(x+h)}{h}-\sum_{i=1}^{n} f_{i}\left(\left(\alpha_{i}+\beta_{i}\right) x+\left(\alpha_{i}+2 \beta_{i}\right) h\right)\right| \leq \varepsilon,
$$

and these two equations give us

$$
\begin{gathered}
\mid \frac{F(x+2 h)-F(x)}{h}-\sum_{i=1}^{n} f_{i}\left(\left(\alpha_{i}+\beta_{i}\right) x+\beta_{i} h\right) \\
-\sum_{i=1}^{n} f_{i}\left(\left(\alpha_{i}+\beta_{i}\right) x+\left(\alpha_{i}+2 \beta_{i}\right) h\right) \mid \leq 2 \varepsilon .
\end{gathered}
$$

On the other hand, taking $x+2 h$ in place of $y$ in (2.2), we get

$$
\left.\mid \frac{F(x+2 h)-F(x)}{2 h}-\sum_{i=1}^{n} f_{i}\left(\left(\alpha_{i}+\beta_{i}\right) x+2 \beta_{i} h\right)\right) \mid \leq \varepsilon .
$$

This, together with (2.4), yields

$$
\begin{aligned}
& \mid \sum_{i=1}^{n} f_{i}\left(\left(\alpha_{i}+\beta_{i}\right) x+\beta_{i} h\right)+\sum_{i=1}^{n} f_{i}\left(\left(\alpha_{i}+\beta_{i}\right) x+\left(\alpha_{i}+2 \beta_{i}\right) h\right) \\
& \left.\quad-2 \sum_{i=1}^{n} f_{i}\left(\left(\alpha_{i}+\beta_{i}\right) x+2 \beta_{i} h\right)\right) \mid \leq 4 \varepsilon .
\end{aligned}
$$

In the next part of the paper we are going to use a result proved by Baker in [2]. For the sake of completeness we shall cite this theorem.

Theorem 2.1. (Baker [2]) Let $V, B$ be real or complex vector spaces and assume that $B$ is a Banach space. Further suppose that the functions $f_{0}, \ldots, f_{m}: V \rightarrow$ $B$ satisfy for all $x, y \in V$

$$
\left\|\sum_{k=0}^{m} f_{k}\left(\alpha_{k} x+\beta_{k} y\right)\right\| \leq \delta
$$

for some $\delta>0$ and scalars $\alpha_{k}, \beta_{k}$ with

$$
\alpha_{j} \beta_{k}-\alpha_{k} \beta_{j} \neq 0
$$

whenever $j \neq k$. Then for each $k \in\{0, \ldots, m\}$

$$
\left\|\Delta_{h_{m}} \cdots \Delta_{h_{1}} f_{k}(x)\right\| \leq 2^{m} \delta \quad \text { for all } x, h_{1}, \ldots, h_{m} \in V
$$


and there exists a polynomial function $p_{k}: V \rightarrow B$ of order at most $m-1$ and a constant $c_{k}$ such that

$$
\left\|f_{k}(x)-c_{k}-p_{k}(x)\right\| \leq 2^{m+1} \delta \quad \text { for all } x \in V .
$$

Moreover

$$
\sum_{k=0}^{m} p_{k}\left(\alpha_{k} x+\beta_{k} y\right)=0 .
$$

Although Lemma 1 was stated for Eq. (2.1), in the remaining part of the paper we shall work with (1.1) with an additional assumption that $\alpha_{k}+\beta_{k}=1$. There are several reasons to restrict ourselves to this case. Our equations stem from numerical integration and quadratures used to approximate the integral take exactly the form used in (1.1) (with $\alpha_{k}+\beta_{k}=1$ ). Further if we want to prove that functions satisfying our equations are continuous we have to make some assumptions of this kind.

Now we shall state the main result of the paper.

Theorem 2.2. Let $n \in \mathbb{N}$ and let functions $F, f: \mathbb{R} \rightarrow \mathbb{R}$ satisfy, for all $x \neq y$, the inequality

$$
\left.\mid \frac{F(y)-F(x)}{y-x}-\sum_{i=1}^{n} a_{i} f\left(\alpha_{i} x+\left(1-\alpha_{i}\right) y\right)\right) \mid \leq \varepsilon
$$

with some $a_{i} \in \mathbb{R} \backslash\{0\}$ and pairwise distinct $\alpha_{i} \in[0,1], i=1, \ldots, n$. Then there exist a constant $M>0$ and a polynomial function $p$ of order at most $3 n-2$ such that

$$
|f(x)-p(x)|<M \varepsilon .
$$

Further there exist a polynomial $P$ of degree at most $3 n-1$ and a constant $K>0$ such that

$$
|F(x)-P(x)| \leq K \varepsilon,
$$

the function $x \mapsto(F(x)-P(x))$ is Lipschitz continuous and functions $P, p$ satisfy (1.1).

Finally, if $a_{1}+\cdots+a_{n} \neq 0$ then also $f$ must be continuous.

Proof. Using Lemma 1, we can see that $f$ satisfies

$$
\begin{aligned}
& \mid \sum_{i=1}^{n} a_{i} f\left(x+\left(1-\alpha_{i}\right) h\right)+\sum_{i=1}^{n} a_{i} f\left(x+\left(2-\alpha_{i}\right) h\right) \\
& \quad-2 \sum_{i=1}^{n} a_{i} f\left(x+\left(2-2 \alpha_{i}\right) h\right) \mid \leq 4 \varepsilon .
\end{aligned}
$$

Values of $f$ are here calculated at the points of the form $x+\gamma h$ for some values of $\gamma$. First we add these values of $f$ occurring in (2.8) which are calculated at 
the same point. As a result we obtain a sum of a similar form as in (2.8) but with different coefficients of $h$. This means that now we have

$$
\left|\sum_{i=1}^{l} b_{i} f\left(x+\gamma_{i} h\right)\right| \leq 4 \varepsilon
$$

for some $l \in \mathbb{N}, l \leq 3 n$, some $b_{i} \in \mathbb{R}$ and some $\gamma_{i} \in \mathbb{R}$ where $\gamma_{i} \neq \gamma_{j}, i \neq j$. Consequently, condition (2.5) is satisfied. We only have to check that not all numbers $b_{i}$ are zero.

To this end, we observe that points of the form $x+\left(1-\alpha_{i}\right) h$ belong to the interval $[x, x+h]$ and that points $x+\left(2-\alpha_{i}\right) h$ are in $[x+h, x+2 h]$.

First we consider the case $n=1$. In this case inequality (2.8) contains values of $f$ at the points: $x+\left(1-\alpha_{1}\right) h, x+\left(2-\alpha_{1}\right) h$, and $x+\left(2-2 \alpha_{1}\right) h$. At least one of these points is different from the others. Thus its term cannot vanish after our simplification. Further if $n=2$ and at least one of the numbers $\alpha_{1}, \alpha_{2}$ is different from 0 and 1 then we have four different points of the forms $x+\left(1-\alpha_{i}\right) h, x+\left(2-\alpha_{i}\right) h$, and only two of the shape $x+\left(2-2 \alpha_{1}\right) h$. Like before, it means that some of the values of $f$ from (2.8) do not vanish. In the case: $\alpha_{1}=0, \alpha_{2}=1$ we have a concrete form of (2.8) and it is easy to check that the left-hand side of this inequality is nontrivial. To finish this part of the proof assume that $n \geq 3$. In this case the system

$$
x+\left(1-\alpha_{1}\right) h, \ldots, x+\left(1-\alpha_{n}\right) h, x+\left(2-\alpha_{1}\right) h, \ldots, x+\left(2-\alpha_{n}\right) h
$$

contains at least $2 n-2$ different points. Thus there must be an $i_{0} \in\{1, \ldots, n\}$ such that

$$
x+\left(1-\alpha_{i_{0}}\right) h \neq x+\left(2-2 \alpha_{i}\right) h \quad \text { for all } i=1, \ldots, n
$$

or

$$
x+\left(2-\alpha_{i_{0}}\right) h \neq x+\left(2-2 \alpha_{i}\right) h \quad \text { for all } i=1, \ldots, n .
$$

In view of Theorem 2.1, this means that some function $a_{i_{0}} f$ is close to a polynomial function. Thus there exists $p(x)=p_{0}+p_{1}(x)+\cdots+p_{3 n-2}(x)$ such that functions $p_{k}$ are monomial of orders $k$ and the inequality

$$
|f(x)-p(x)| \leq M \varepsilon
$$

is satisfied with some $M>0$. Therefore we may write

$$
f(x)=p_{0}+p_{1}(x)+\cdots+p_{3 n-2}(x)+r(x)
$$

where $|r(x)| \leq M \varepsilon$.

Now we shall use this equality in (2.6). Without loss of generality we may assume that $F(0)=0$ thus, taking $y=0$ in (2.6), we may write

$$
\left|\frac{F(x)}{x}-\sum_{i=1}^{n} a_{i}\left(p_{0}+p_{1}\left(\alpha_{i} x\right)+\cdots+p_{3 n-2}\left(\alpha_{i} x\right)+r\left(\alpha_{i} x\right)\right)\right| \leq \varepsilon .
$$


Using here the boundedness of $r$, we get for some $K>0$

$$
\left|\frac{F(x)}{x}-\left(P_{0}+P_{1}(x)+\cdots+P_{3 n-2}(x)\right)\right| \leq K \varepsilon
$$

i.e.

$$
F(x)=x\left(P_{0}+P_{1}(x)+\cdots+P_{3 n-2}(x)+R(x)\right),
$$

where $P_{0}=\sum_{i=1}^{n} a_{i} p_{0}$ is a constant, $P_{k}(x)=\sum_{i=1}^{n} a_{i} p_{k}\left(\alpha_{i} x\right)$ is a monomial function of order $k$ and $|R(x)| \leq K \varepsilon$. Using (2.11), (2.10) and the boundedness of $r$ in (2.6), we get for some $L>0$

$$
\begin{aligned}
& \mid \frac{y\left(P_{0}+\sum_{k=1}^{3 n-2} P_{k}(y)+R(y)\right)-x\left(P_{0}+\sum_{k=1}^{3 n-2} P_{k}(x)+R(x)\right)}{y-x} \\
& \quad-\sum_{i=1}^{n} a_{i}\left(p_{0}+p_{1}\left(\alpha_{i} x+\left(1-\alpha_{i}\right) y\right)+\cdots+p_{3 n-2}\left(\alpha_{i} x+\left(1-\alpha_{i}\right) y\right)\right) \mid \leq L \varepsilon .
\end{aligned}
$$

Now we observe that $\frac{y P_{0}-x P_{0}}{y-x}=\sum_{i=1}^{n} a_{i} p_{0}$ which, used in (2.12), gives us

$$
\begin{aligned}
& \mid \frac{y\left(\sum_{k=1}^{3 n-2} P_{k}(y)+R(y)\right)-x\left(\sum_{k=1}^{3 n-2} P_{k}(x)+R(x)\right)}{y-x} \\
& \quad-\sum_{i=1}^{n} a_{i}\left(p_{1}\left(\alpha_{i} x+\left(1-\alpha_{i}\right) y\right)+\cdots+p_{3 n-2}\left(\alpha_{i} x+\left(1-\alpha_{i}\right) y\right)\right) \mid \leq L \varepsilon .
\end{aligned}
$$

In the next step of the proof we substitute $2 x$ and $2 y$ instead of $x$ and $y$, respectively, and we arrive at

$$
\begin{aligned}
& \mid \frac{y\left(\sum_{k=1}^{3 n-2} 2^{k} P_{k}(y)+R(2 y)\right)-x\left(\sum_{k=1}^{3 n-2} 2^{k} P_{k}(x)+R(2 x)\right)}{y-x} \\
& \quad-\sum_{i=1}^{n} a_{i}\left(2 p_{1}\left(\alpha_{i} x+\left(1-\alpha_{i}\right) y\right)+\cdots+2^{3 n-2} p_{3 n-2}\left(\alpha_{i} x+\left(1-\alpha_{i}\right) y\right)\right) \mid \leq L \varepsilon .
\end{aligned}
$$

Dividing both sides of $(2.14)$ by $2^{3 n-2}$, we can see that the only terms which remain unchanged are those of order $3 n-2$, all others are divided by powers of two. If we repeat this operation then all expressions with orders smaller than $3 n-2$ tend to zero. This yields

$$
\frac{y P_{3 n-2}(y)-x P_{3 n-2}(x)}{y-x}=\sum_{i=1}^{n} a_{i} p_{3 n-2}\left(\alpha_{i} x+\left(1-\alpha_{i}\right) y\right) .
$$


Now we may use a result from [5] which states that the mapping $x \mapsto x P_{3 n-2}(x)$ is an ordinary polynomial. This means that $P_{3 n-2}(x)=b_{3 n-1} x^{3 n-1}$, for some real number $b_{3 n-1}$.

Further, using (2.15) in (2.13), we get

$$
\begin{aligned}
& \mid \frac{y\left(\sum_{k=1}^{3 n-3} P_{k}(y)+R(y)\right)-x\left(\sum_{k=1}^{3 n-3} P_{k}(x)+R(x)\right)}{y-x} \\
& \quad-\sum_{i=1}^{n} a_{i}\left(p_{1}\left(\alpha_{i} x+\left(1-\alpha_{i}\right) y\right)+\cdots+p_{3 n-3}\left(\alpha_{i} x+\left(1-\alpha_{i}\right) y\right)\right) \mid \leq L \varepsilon .
\end{aligned}
$$

Repeating this procedure sufficiently many times, we show that $F$ is of the form (2.7). Moreover, we have

$$
\frac{b_{k} y^{k+1}-b_{k} x^{k+1}}{y-x}=\sum_{i=1}^{n} a_{i} p_{k}\left(\alpha_{i} x+\left(1-\alpha_{i}\right) y\right), \quad k=1, \ldots, 3 n-2
$$

i.e

$$
b_{k}\left(y^{k}+y^{k-1} x+\cdots+x^{k}\right)=\sum_{i=1}^{n} a_{i} p_{k}\left(\alpha_{i} x+\left(1-\alpha_{i}\right) y\right),
$$

for all $x \neq y$. Note also that the last inequality which we obtain is of the form

$$
\left|\frac{y R(y)-x R(x)}{y-x}\right| \leq L \varepsilon .
$$

Now let $A$ be a $k$-additive and symmetric function such that

$$
p_{k}(x)=A(\underbrace{x, \ldots, x}_{k}),
$$

then (2.17) may be rewritten in the form

$$
\begin{array}{r}
b_{k}\left(y^{k}+y^{k-1} x+\cdots+x^{k}\right)=\sum_{i=1}^{n} a_{i}\left(A\left(\alpha_{i} x, \ldots, \alpha_{i} x\right)\right. \\
\left.+\cdots+A\left(\left(1-\alpha_{i}\right) y, \ldots,\left(1-\alpha_{i}\right)\right) y\right) .
\end{array}
$$

Now, let $q_{j}$ be a sequence of rational numbers tending to 1 and different from 1. Then, taking $y=q_{j} x$ in (2.18), we get

$$
\begin{array}{r}
b_{k} x^{k}\left(q_{j}^{k}+\cdots+1\right)=\sum_{i=1}^{n} a_{i}\left(A\left(\alpha_{i} x, \ldots, \alpha_{i} x\right)\right. \\
\left.+\cdots+q_{j}^{k} A\left(\left(1-\alpha_{i}\right) x, \ldots,\left(1-\alpha_{i}\right)\right) x\right) .
\end{array}
$$


Tending here with $j$ to infinity, we get

$$
k b_{k} x^{k}=\sum_{i=1}^{n} a_{i} p_{k}(x)
$$

thus also $p_{k}$ is continuous, provided that $\sum_{i=1}^{n} a_{i} \neq 0$.

Remark 1. A careful inspection of the proof of Theorem 2.2 shows that it is possible to obtain the exact values of $M$ and $K$ but the formulas expressing them would be very complicated. Moreover these values rely strongly on our method and, therefore, they are probably far from being optimal.

The following corollary will show that the degrees of $f$ and $F$ obtained in Theorem 2.2 may, in the case of concrete equations be lower. The inequality considered in this corollary is motivated by the Simpson quadrature rule.

Corollary 1. If functions $f, F$ satisfy the inequality

$$
\left|\frac{F(y)-F(x)}{y-x}-\left(\frac{1}{6} f(x)+\frac{2}{3} f\left(\frac{x+y}{2}\right)+\frac{1}{6} f(y)\right)\right| \leq \varepsilon
$$

then there exist $a, b, c, d \in \mathbb{R}$ and $M, K>0$ such that

$$
F(x)=a x^{3}+b x^{2}+c x+d+R(x)
$$

and

$$
f(x)=3 a x^{2}+2 b x+c+r(x),
$$

where $|r(x)| \leq M \varepsilon,|R(x)| \leq K \varepsilon$ and

$$
\left|\frac{y R(y)-x R(x)}{y-x}\right| \leq \varepsilon .
$$

Proof. Using Theorem 2.2 we can see that there exist functions $r$ and $R$ such that

$$
F(x)=P(x)+R(x)
$$

where $P$ is a polynomial of degree at most 5 and

$$
f(x)=p(x)+r(x)
$$

where $p$ is a polynomial of degree at most 4. However from Theorem 2.2 we know that functions $P, p$ satisfy the equation

$$
\frac{P(y)-P(x)}{y-x}=\frac{1}{6} p(x)+\frac{2}{3} p\left(\frac{x+y}{2}\right)+\frac{1}{6} p(y) .
$$

It is well known (see for example [7]) that in such a case the degree of $p$ is not greater than 3. Further using the continuity of $p$ and tending with $y$ to $x$, we get that $P^{\prime}=p$, as claimed.

The next example will show that it is impossible to get the superstability result (as it was the case in the paper [10]). 
Example. Assume that $\sum_{i=1}^{n} a_{i}=1$, take function $F$ as any function satisfying the Lipschitz condition with constant $\varepsilon / 2$ and $f$ as a function bounded by $\varepsilon / 2$. Then functions $F, f$ satisfy inequality (2.6).

Remark 2. As it is easy to observe, inequalities considered in [10] and in the present paper have a joint generalization which is given by

$$
\left|F(y)-F(x)-(y-x) \sum_{i=1}^{n} a_{i} f\left(\alpha_{i} x+\beta_{i} y\right)\right| \leq \varepsilon|x-y|^{p} .
$$

In view of results contained in [10] and of Theorem 2.2, we may say that the stability problem posed in this way has a satisfactory solution for $p=1$ and a partial solution for $p=0$.

Open Access. This article is distributed under the terms of the Creative Commons Attribution 4.0 International License (http://creativecommons.org/licenses/by/4.0/), which permits unrestricted use, distribution, and reproduction in any medium, provided you give appropriate credit to the original author(s) and the source, provide a link to the Creative Commons license, and indicate if changes were made.

\section{References}

[1] Aczél, J.: A mean value property of the derivative of quadratic polynomials - without mean values and derivatives. Math. Mag. 58(1), 42-45 (1985)

[2] Baker, J.A.: A general functional equation and its stability. Proc. Am. Math. Soc. 133(6), 1657-1664 (2005)

[3] Kannappan, Pl., Riedel, T., Sahoo, P.K.: On a functional equation associated with Simpson's rule. Results Math. 31(1-2), 115-126 (1997)

[4] Koclęga-Kulpa, B., Szostok, T., Wąsowicz, Sz.: On functional equations connected with quadrature rules. Georgian Math. J. 16(4), 725-736 (2009)

[5] Koclęga-Kulpa, B., Szostok, T.: On a class of equations stemming from various quadrature rules. Acta Math. Hung. 130(4), 340-348 (2011)

[6] Lisak, A., Sablik, M.: Trapezoidal rule revisited. Bull. Inst. Math. Acad. Sin. (N.S.) 6(3), 347-360 (2011)

[7] Sahoo, P.K., Riedel, T.: Mean Value Theorems and Functional Equations. World Scientific, Singapore (1998)

[8] Sahoo, P.K.: On a functional equation associated with the trapezoidal rule. Bull. Inst. Math. Acad. Sin. (N.S.) 2(1), 67-82 (2007)

[9] Sahoo, P.K.: On a functional equation associated with the trapezoidal rule II. Bull. Inst. Math. Acad. Sin. (N.S.) 4(1), 9-23 (2009)

[10] Szostok, T., Wąsowicz, Sz.: On the stability of the equation stemming from Lagrange MVT. Appl. Math. Lett. 24(4), 541-544 (2011) 
Tomasz Szostok

Institute of Mathematics

University of Silesia

Bankowa 14

40-007 Katowice

Poland

e-mail: tszostok@math.us.edu.pl

Received: March 31, 2015

Revised: February 8, 2016 